achieved by manual plotting, in terms of speed, accuracy, and immediacy and comprehensiveness of the information displayed.

Commander Clissold's proposed method of reducing the danger is the provision of an extra man on the bridge, charged with the duty of plotting continuously, i.e. not merely regularly recording the successive positions of targets, but also regularly constructing and revising relevant vector triangles. I suggest that the self-plotting radar now available, which at the push of a button brings up on the main radar screen continuously up-dated, high integrity 4-plot tracks of all targets, in true motion, actual relative motion or relative motion predicted for a proposed manœuvre by own ship, is in practice a substantially greater asset to safety. It is also one which may well be more easily and economically provided.

\title{
A Manœuvring Diagram for Avoiding Collisions at Sea
}

\section{Captain A. N. Cockcroft}

INTRODUCTION. In 1970 the Institute formed a Working Party to formulate views on the revision of the collision regulations. Its Interim Report is published in the Journal for October 1970 and two further contributions, by Calvert and García-Frías, were published in a subsequent number (24, 41 3-20). The Interim Report referred to the value that a manouvring diagram along the lines originally proposed by Calvert and Hollingdale would have in helping the mariner to assess the effects of collision situations on alterations of course and speed. It has not been possible to secure unanimity about the form of this diagram, but the one illustrated below is based on the contributions of several members and has met with the approval of the majority of the Working Party.

PRINCIPLES AND CONCEPTS. (I) Manœurring guidance is likely to be of greatest value when navigating with radar in restricted visibility. In such conditions bold alterations of course and speed are necessary if the action is to be readily detected by other vessels. The recommended alterations are in accordance with helm action normally taken by power-driven vessels under the Steering and Sailing Rules, but smaller alterations are usually sufficient for visual situations. For greater compatibility with the in-sight rules it would be desirable to allow earlier action by the privileged vessel than is permitted under the existing Rule 2 I.

(2) The diagram is restricted to course alterations. Advice on speed changes is given in notes accompanying the diagram. It is considered that reductions of speed should not be prohibited for any sector in poor visibility and, as vessels generally proceed at the greatest speed compatible with safety, appreciable increases of speed are usually either dangerous or impossible.

(3) Course alterations which will cause anti-clockwise rotation of the sightline if the other vessel keeps her course and speed are recommended, except for the port beam sector where they would be ineffective. Turns which would cause clockwise rotation but maximum disengagement are recommended as a 
form of emergency action when a vessel approaching from near the port beam fails to keep out of the way. Such action would not complement subsequent positive action which might be taken by the other vessel, but the recommendation to keep the threat astern should be sufficient to prevent a new collision situation developing.

(4) The onus to manœuvre is placed on an overtaking vessel and a crossing vessel approaching from near the port beam of another vessel. Such vessels will have greater capability for positive avoiding action. When a vessel is observed to be approaching from within two points of the port beam, or from more than two points abaft the beam, it is recommended that action should only be taken at short range when the other vessel has failed to keep clear.

(5) A small number of easily comprehended general recommendations for helm action are indicated on the diagram, rather than specific turns for different bearings. This approach gives some flexibility. As changes of speed are also included in the system, application is not restricted to open water situations.

(6) Crossing situations which are initially negative are catered for by recommending the vessel which has the other on her own starboard side to turn to starboard until the bearing is appreciably to port and to keep the bearing to port when resuming course. The vessel with the other on her own port bow is recommended to reduce speed without changing course in a close negative situation, but to resume normal speed on detecting a starboard turn by the other vessel. If recommended action does not resolve the initial situation the diagram can be used to indicate further helm action for the new direction of threat. A capacity to exchange signals so as to agree on complementary manœuvres would, of course, be desirable; but there appears to be little prospect of satisfactorily achieving this in the near future.

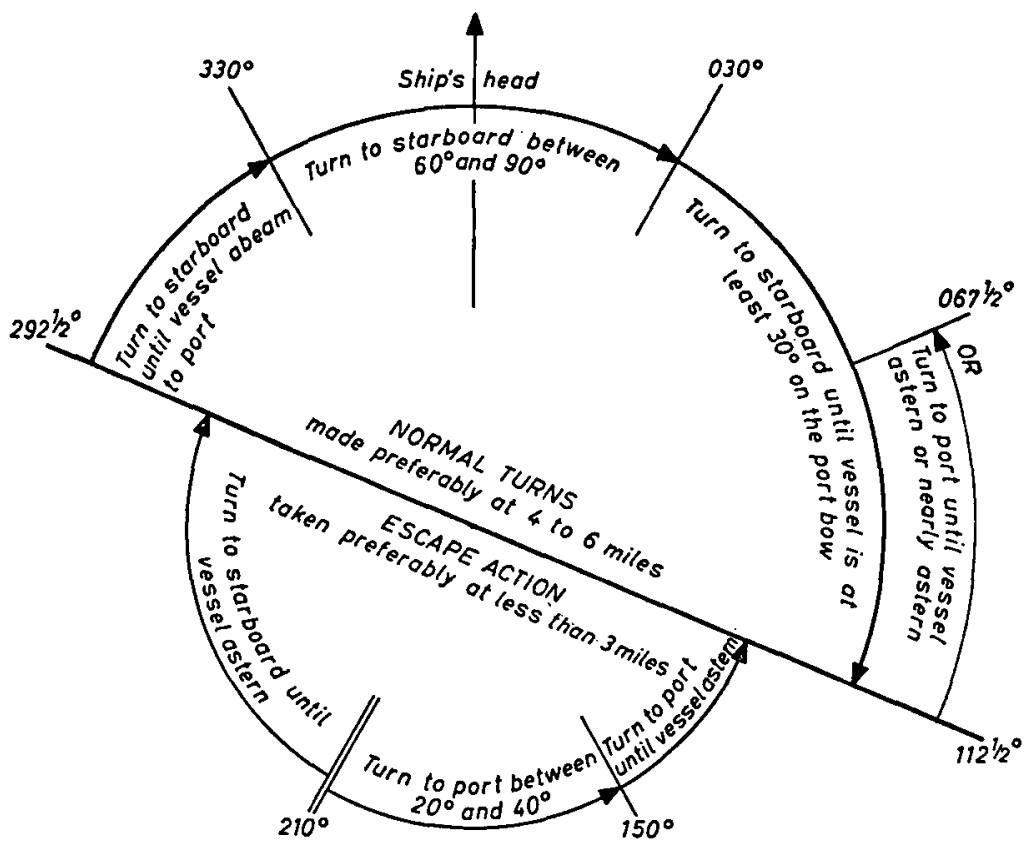

FIG. 1. Manœuvring diagram 
Note: Positive action is an alteration of course and/or speed which causes an anti-clockwise rotation of the sight-line between the two vessels if the other vessels stands-on. Negative action causes the sight-line to rotate in a clockwise direction.

Resumption of course. After turning to starboard for a vessel on the starboard side keep the vessel to port when resuming course.

Escape action. A vessel approaching from the port side and rear sector can normally be expected to take early avoiding action. The suggested turns are recommended for use when such a vessel fails to keep out of the way. As an alteration to put the bearing astern may not complement subsequent action by the other vessel, it is recommended that further turns be made to keep the vessel astern until she is well clear.

SPEED ChANGes IN Restricted visibility. Reductions of speed. A vessel can reduce speed or stop at any time and such action is recommended when the compass bearing of a vessel on the port bow is gradually changing in a clockwise direction (increasing). A reduction of speed should be made as an alternative to, and not in conjunction with, the suggested turn to starboard for avoiding a vessel either on the port bow or ahead. Normal speed should be resumed if it becomes apparent that a vessel on the port side has either subsequently turned to starboard in order to pass astern, or stopped.

Increases of speed. It will sometimes be advantageous to increase speed if this is possible within the limitations of the requirement to proceed at a safe speed. An increase of speed may be appropriate when the vessel to be avoided is astern, or on the port quarter, or near the port beam, either initially or after taking the alter course action indicated in the diagram.

Limitations. The presence of other vessels and/or lack of sea room may impose limitations on the manœurres which can be made, but it should be kept in mind that small changes of course and/or speed are unlikely to be detected by radar.

Caution. It is essential to ensure that any action taken is having the desired effect. If not, the recommended turns can normally be applied successively for newly developed collision situations with the same vessel.

\section{Historical Hyperbolae}

\section{Claud Powell}

Ar a first glance, one of the two accompanying diagrams might be taken to represent a three-station Decca or Loran chain, while the other offers familiar instruction on how to interpolate between the lattice lines and plot the fix. In fact, these are illustrations in a German Army handbook entitled An Introduction to Sound Ranging and the Work of the Sound Ranging Section, published in July 19 I 8.

$\mathrm{A}, \mathrm{B}$ and $\mathrm{C}$ are interconnected receiving stations (microphones) on baselines of about $\mathrm{I} \mathrm{km}$. and their object is to find the position of a hostile gun by comparing the times at which the sound of its report arrives at the three known positions. Assuming a constant propagation speed, comparing the three observations yields a pair of hyperbolic position lines whose intersection gives the gun's position. As in Loran the lattice lines are numbered directly in units of time difference 